\title{
Production of ultracold neutrons from a cold neutron beam on a ${ }^{2} \mathrm{H}_{2}$ target
}

F. Atchison, B. van den Brandt, T. Bryś, ${ }^{*}$ M. Daum, P. Fierlinger, ${ }^{\dagger}$ P. Hautle, R. Henneck, S. Heule, ${ }^{\dagger}$ M. Kasprzak, ${ }^{\ddagger}$ K. Kirch, ${ }^{\S}$ J. A. Konter, A. Michels, A. Pichlmaier, M. Wohlmuther, and A. Wokaun* Paul Scherrer Institut, CH-5232 Villigen PSI, Switzerland ${ }^{\|}$

\author{
K. Bodek and U. Szerer" \\ Institute of Physics, Jagiellonian University, Cracow, Poland
}

P. Geltenbort

Institut Laue-Langevin, Grenoble, France

J. Zmeskal

Stefan Meyer Institut für subatomare Physik, Austrian Academy of Sciences, Vienna, Austria

Y. Pokotilovskiy

Joint Institute for Nuclear Research, Dubna, Russia

(Received 4 January 2005; published 20 May 2005)

\begin{abstract}
The production rates of ultracold neutrons (UCN) from cold neutrons on gaseous, liquid, and solid deuterium targets have been measured. The comparison of the measured and calculated UCN production on gaseous ${ }^{2} \mathrm{H}_{2}$ is used to calibrate the simulated target extraction and transport efficiencies of the experimental apparatus. The production cross section in solid ${ }^{2} \mathrm{H}_{2}$ at $8 \mathrm{~K}$ for UCN with energies between 0 and $250 \mathrm{neV}$ is $R_{\text {solid, } 8 \mathrm{~K}}=$ $\sigma_{\text {solid } 8 \mathrm{~K}}^{\mathrm{CN} \rightarrow \mathrm{UCN}} \rho=(1.11 \pm 0.23) \times 10^{-8} \mathrm{~cm}^{-1}$. This value is consistent with other experiments in which UCN had been extracted from ${ }^{2} \mathrm{H}_{2}$. The value also agrees with calculations using the incoherent approximation and a simple Debye model and corroborates predictions for UCN densities expected at the high-intensity UCN source at the Paul Scherrer Institut. The temperature dependence of the UCN production in solid ${ }^{2} \mathrm{H}_{2}$ down to $8 \mathrm{~K}$ can be explained within the same model when multiple-phonon excitation is included.
\end{abstract}

DOI: 10.1103/PhysRevC.71.054601

PACS number(s): 28.20.Gd, 28.20.Cz, 25.40.Fq

\section{INTRODUCTION}

Ultracold neutrons (UCN) have energies low enough that they are totally reflected by suitable materials and so can be confined in bottles. They are predominantly used in fundamental physics experiments, e.g., in the search for an electric dipole moment and measurement of a lifetime, that require low velocities and long interaction and observation times, e.g. $[1,2]$. At the present time, the accuracy of such measurements is limited mainly by statistics [3], ${ }^{1}$ and significantly higher UCN densities will allow more stringent tests of the standard

\footnotetext{
*Also at Institute for Technical Chemistry, ETH Zürich, Switzerland.

${ }^{\dagger}$ Also at Physik Institut, University of Zürich, Switzerland.

${ }^{\ddagger}$ Also at Stefan Meyer Institut für subatomare Physik, Austrian Academy of Sciences, Vienna, Austria.

${ }^{\S}$ Corresponding author, klaus.kirch@psi.ch

"URL: http://ucn.web.psi.ch

`During summer 2004 at Paul Scherrer Institut, CH-5232 Villigen PSI, Switzerland.

${ }^{1}$ The highest UCN intensity worldwide is currently available at Institut Lane-Langevin, Grenoble [3].
}

model. Several projects aim at building new sources for UCN in order to provide the required increase in intensity. Two main source types having the potential to produce substantially higher UCN densities are being pursued based on using either superfluid helium [4-7] or solid deuterium to down scatter (convert) cold neutrons [8-13].

A spallation neutron source dedicated to UCN production and based on solid ${ }^{2} \mathrm{H}_{2}$ is presently under construction at the Paul Scherrer Institut (PSI) in Switzerland [14]; fast neutrons produced by spallation are thermalized in heavy water and finally cooled and converted to UCN by the solid ${ }^{2} \mathrm{H}_{2}$. The $\mathrm{UCN}$ are produced during a several seconds long pulse of a $2 \mathrm{~mA} 590 \mathrm{MeV}$ proton beam and collected in a storage vessel from which they are distributed to experiments.

An associated research program aimed at providing missing pieces of information concerning UCN source physics is in progress. In relation to influencing the UCN density achievable, particular concerns are the UCN production in and the extraction from solid ${ }^{2} \mathrm{H}_{2}$. The transmission of UCN through solid ${ }^{2} \mathrm{H}_{2}$ prepared in various ways as well as through gaseous and liquid ${ }^{2} \mathrm{H}_{2}$ has been measured recently $[15,16]$, and the results help with understanding the extraction process. The work described in the present paper is concerned with the study of UCN production inside solid ${ }^{2} \mathrm{H}_{2}$. 


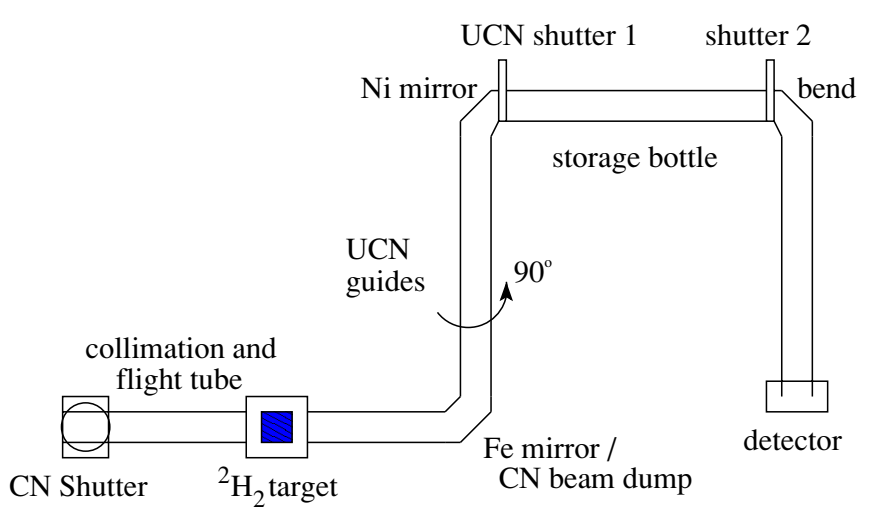

FIG. 1. (Color online) Schematical setup (not to scale): The CN beam of diameter $40 \mathrm{~mm}$ is collimated over a length of about $1.5 \mathrm{~m}$ before hitting the ${ }^{2} \mathrm{H}_{2}$ target sitting behind a $38 \mathrm{~mm}$ aperture. UCN can leave the target and reach the guide system. A mirror reflects UCN upward where they can reach the storage bottle at about $1 \mathrm{~m}$ height after being reflected by a second mirror. Two UCN shutters allow storage measurements. The UCN setup is rotated by $90^{\circ}$ around the vertical $\mathrm{UCN}$ guide, moving the detector out of the $\mathrm{CN}$ beam axis.

\section{EXPERIMENTAL ARRANGEMENT}

\section{A. General considerations}

The aim of the experiment is to measure the UCN production rate from cold neutrons interacting in solid ${ }^{2} \mathrm{H}_{2}$. This has been achieved by using a UCN detector with a well-defined geometry and a normalized simulation to calculate the detection efficiency. To expedite this process, results for both liquid and gaseous ${ }^{2} \mathrm{H}_{2}$ have also been taken during the experiment.

The layout of the experimental apparatus is shown in Fig. 1. A well-collimated beam of cold neutrons $(\mathrm{CN})$ is directed onto the target cell containing the solid ${ }^{2} \mathrm{H}_{2}$. After the cell, a guide system with mirrors transports and deflects neutrons in the $\mathrm{UCN}$ energy region vertically to a UCN bottle about $1 \mathrm{~m}$ above the solid ${ }^{2} \mathrm{H}_{2}$ target (energy difference is about $100 \mathrm{neV}$ ). The bottle is made of Be-coated quartz ${ }^{2}$ and can store neutrons with energies up to about $250 \mathrm{neV}$; it is equipped with two shutters [coated with diamond-like carbon (DLC), ${ }^{3}$ which can totally reflect up to essentially the same UCN energy] so that it can be opened and closed at either end.

The experiment was mounted on the polarized cold neutron beam line for fundamental physics (FUNSPIN) [17] at the Swiss spallation neutron source SINQ. The flux spectrum at the entrance to the experiment (shown in Fig. 2) was converted from the measured wavelength neutron density spectrum of [17]. The integrated neutron flux incident on the target cell was measured in situ using gold foil activation and is

$$
\Phi_{\mathrm{CN}}=(4.5 \pm 1.0) \times 10^{7} \mathrm{~cm}^{-2} \mathrm{~s}^{-1} \mathrm{~mA}^{-1},
$$

\footnotetext{
${ }^{2}$ The Be-coated tube was obtained from the Petersburg Nuclear Physics Institute, Gatchina, Russia.

${ }^{3}$ The DLC-coated foils were obtained from the Fraunhofer Institute, Dresden, Germany.
}

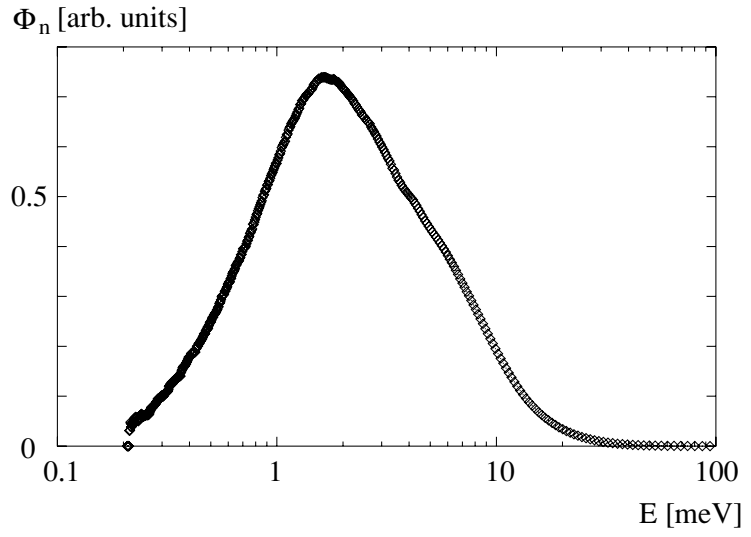

FIG. 2. Cold neutron flux vs neutron energy at the exit of the FUNSPIN neutron guide [17].

where the milliampere refers to the proton beam current incident at the SINQ spallation target. The relatively large uncertainty in the measurement is mainly due to imprecision in the mass of the irradiated part of the foil and the need to use a foil of nonstandard shape for the analysis machine.

The experiment may be run in two ways: "storage" mode (SM) and "flow-through" mode (FM). In the storage mode, the UCN bottle is filled to equilibrium. At the start of the measurement, it is open to the solid ${ }^{2} \mathrm{H}_{2}$ target $(\mathrm{CN}$ shutter and UCN shutter 1 open and UCN shutter 2 closed). After a suitable time interval that allows UCN in the bottle to reach equilibrium density, shutter 1 and the $\mathrm{CN}$ shutter are closed. After a few more seconds (enough time to let the non-UCN leave the bottle), the number of neutrons inside the bottle are counted by opening shutter 2 and letting them reach the detector. This method gives a direct and unbiased measurement of the UCN produced by the system.

In the flow-through mode, the UCN spectrum passed is slightly wider than that in a Be storage bottle (critical velocity $6.9 \mathrm{~m} / \mathrm{s}$ ) but can be related to it by calculation. Here, this mode is the preferred method of making the actual production rate measurements. In this method, all shutters are open and the guide system following the ${ }^{2} \mathrm{H}_{2}$ target acts as a UCN spectrometer.

The effectiveness of the spectrometer was proven by observing that the count rate in the detector dropped from about $0.4 \mathrm{~s}^{-1}$ with the solid ${ }^{2} \mathrm{H}_{2}$ target to about $0.010-0.015 \mathrm{~s}^{-1}$ (for the $1.2 \mathrm{~mA}$ proton beam onto the SINQ target) independently of whether either or both the UCN shutters were closed. The same (background) rate was observed for the empty target and all shutters open. This background rate is believed to be due to fast neutrons; it drops to $0.002 \mathrm{~s}^{-1}$ with the main beam line shutter closed and to $0.001 \mathrm{~s}^{-1}$ without protons onto the SINQ target.

\section{B. Details}

Again referring to Fig. 1, $\mathrm{CN}$ enter the experiment through a $40 \mathrm{~mm}$ long, $42 \mathrm{~mm}$ diameter $\mathrm{Pb}$ collimator, clad with a $1 \mathrm{~mm}$ thick layer of ${ }^{6} \mathrm{LiF}$ polymer, and a pneumatically driven 


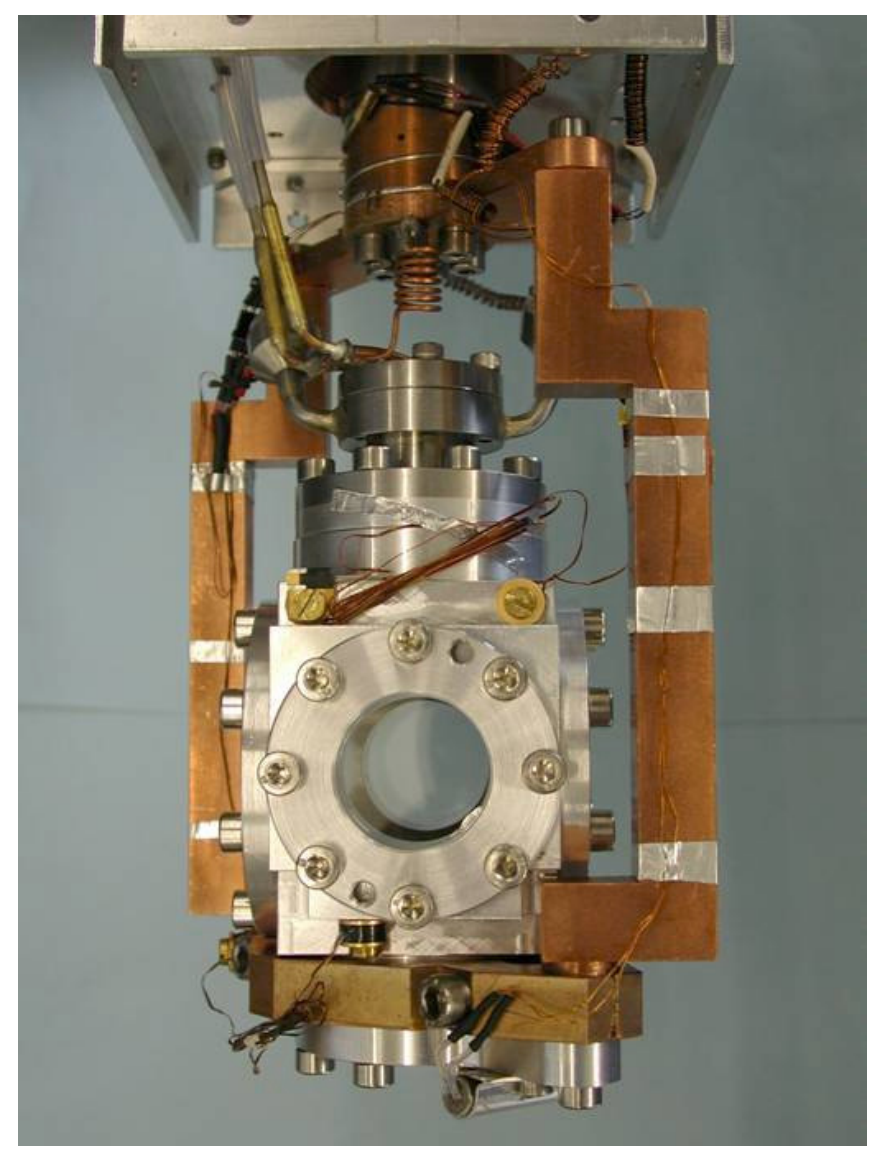

FIG. 3. (Color online) Sideview of the Al target cell mounted on the He-flow cryostat. The optical windows allow inspection of the full target volume accessible to the neutron beam. Three thermometers and two heaters are installed on the target for the control of the solidification and cooling process. The $80 \mathrm{~K}$ thermal shield has been removed for the photograph. The neutron beam axis is horizontal and perpendicular to the optical axis. For details, see [18].

rotating shutter. Immediately after the shutter, the neutrons enter a vacuum flight tube through a $100 \mu \mathrm{m} \mathrm{Zr}$ window. The final collimation of the $\mathrm{CN}$ beam is made with two elements: (i) a $42 \mathrm{~mm}$ aperture $\mathrm{Cd}-\mathrm{Pb}$ sandwich in front of the cryostat chamber and (ii) a $38 \mathrm{~mm}$ aperture Cd element, defining the final beam spot, mounted on the $80 \mathrm{~K}$ thermal shield of the target cell.

The $\mathrm{CN}$ enter the target cell through a $150 \mu \mathrm{m}$ thick AlMg3 (AA5754) window, which is Ni coated on the inside for UCN reflection. The target cell (see Fig. 3) is similar to one used earlier [18] but $40 \mathrm{~mm}$ long in the neutron beam direction. The target cell is mounted on a helium flow cryostat and connected to a ${ }^{2} \mathrm{H}_{2}$ gas system [18]. The ${ }^{2} \mathrm{H}_{2}$ purity was $99.95 \%$ with the remainder potentially being $\mathrm{H}^{2} \mathrm{H}$. The molecular configuration was measured by Raman spectroscopy and found to be $(98.5 \pm 0.5) \%$ in the ortho state $(S=0,2 ; J=0)$. The ${ }^{2} \mathrm{H}_{2}$ pressure in the system at room temperature was $1330 \mathrm{hPa}$, and the total volume of the gas system was about $80 \mathrm{dm}^{3}$. The exit window of the target cell is $150 \mu \mathrm{m} \mathrm{AlMg} 3$ (without Ni coating). A short section of $45 \mathrm{~mm}$ inner diameter UCN guide (Ni-coated $\mathrm{Al}$ ) starts $0.5 \mathrm{~mm}$ after this window and ends
$0.5 \mathrm{~mm}$ in front of a $16 \mu \mathrm{m} \mathrm{Al}$ foil separating the cryostat and external UCN guide vacuum.

The external UCN guide system has a $56 \mathrm{~mm}$ inner diameter and is made from stainless steel chemically coated with $\mathrm{Ni}$; the nickel layers made by this process contain several percent of phosphorous and are nonmagnetic. UCN are reflected upward by a mirror set at $45^{\circ}$ mounted about $70 \mathrm{~cm}$ from the target cell. The mirror is made from a $10 \mathrm{~cm}$ diameter $\mathrm{Si}$ wafer coated with $110 \mathrm{~nm}$ of iron. A ${ }^{6} \mathrm{LiF}$ polymer slab is placed immediately behind this mirror to act as a beam dump for the through-going $\mathrm{CN}$. The iron layer can be magnetized for polarization measurements. A second $45^{\circ}$ mirror, Ni-coated silicon in this case, is mounted about $1 \mathrm{~m}$ above the first to bring the beam back to the horizontal. After the second mirror, a $10 \mathrm{~cm}$ long guide connects via a shutter to the storage bottle. The shutter consists of a $15 \mathrm{~mm}$ thick sliding plastic sheet between stainless steel plates. In the open position, a $15 \mathrm{~mm}$ long piece of UCN guide tube is moved into the neutron path; in the closed position, the shutter is the lid of the neutron bottle and the inner side is covered with a $100 \mu \mathrm{m}$ Polyethylene Terephthalate (PET) foil coated with DLC. There is a gap of about $0.2 \mathrm{~mm}$ between the storage bottle and the shutter. The storage bottle is a Be-coated quartz tube with $65 \mathrm{~mm}$ inner diameter and $108 \mathrm{~cm}$ length. The storage bottle is closed on the second side by a second shutter similar to the first. In the closed position, the DLC-coated PET foil is self-supporting, so relatively fast neutrons could be transmitted. On the exit side, the second shutter connects via a bend and another $80 \mathrm{~cm}$ long guide down to the UCN detector. The detector is a gas counter ${ }^{4}$ with $18 \mathrm{hPa}{ }^{3} \mathrm{He}$ and $10 \mathrm{hPa} \mathrm{CO}$ in about $1100 \mathrm{hPa} \mathrm{Ar}$; it is well shielded with a layer of $\mathrm{Cd}$ close to the detector and the final UCN guide, layers of borated plastic, and more than $0.5 \mathrm{~m}$ of paraffin blocks. The shielding behind the detector is somewhat weaker because of limited space in the area and the $1 \mathrm{~m}$ of concrete support below it.

\section{Detection efficiency}

To extract production cross sections from the measured data, we need to deconvolute the overall efficiency function for the UCN spectrometer, including the coupling to the target cell. Presently there is insufficient detailed knowledge to be able to estimate this directly; for instance, we do not know what ratio of diffuse to specular reflection at the guide walls to use or the velocity distribution of the neutrons escaping the target cell. However, these difficulties are by-passed by using results measured for gaseous ${ }^{2} \mathrm{H}_{2}$ to normalize a detailed simulation of the spectrometer. In the case of gas, the scatterer density is low so that the UCN production in the target cell will be closely spatially homogenous. Also, recent experimental results [15] show very good agreement with the model of Young and Koppel (YK) $[19,20]$ for neutron energies down to the UCN region. That is, in the case with gas in the target cell, the main open parameters are reduced to the amount of diffuse scattering and the efficiency of the gas detector (this will include extra

\footnotetext{
${ }^{4}$ The UCN detector was obtained from the Joint Institute for Nuclear Research, Dubna, Russia.
} 


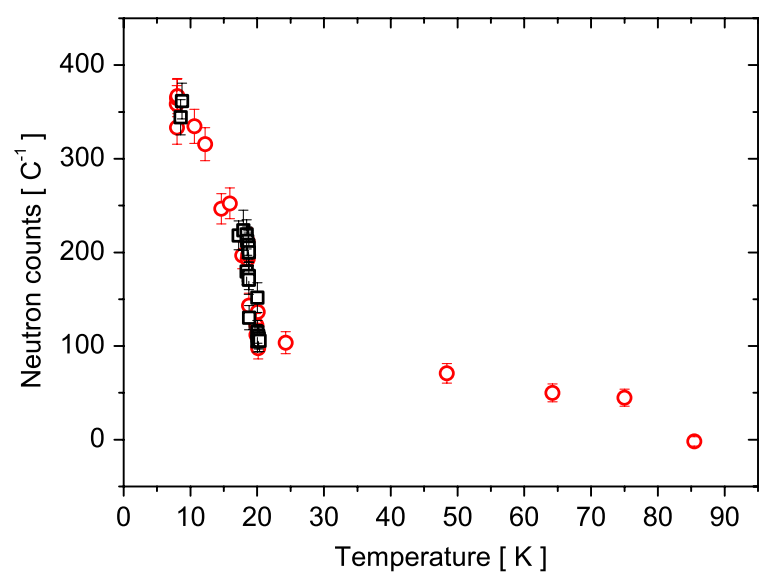

FIG. 4. (Color online) Number of neutrons detected in flow-through mode per $1 \mathrm{C}$ of proton charge onto the SINQ target as a function of ${ }^{2} \mathrm{H}_{2}$ temperature. The data have been corrected for background. The measurement at $85 \mathrm{~K}$ was with an empty cell to confirm the background correction. One data point typically required $1000 \mathrm{~s}$ with $1.2 \mathrm{~mA}$ onto the SINQ target.

losses arising from system imperfections). The ${ }^{2} \mathrm{H}_{2}$ gas results may be used to set values for these, which can then be used for the liquid and solid ${ }^{2} \mathrm{H}_{2}$ results. Although, in truth, we cannot obtain unique values for these two parameters, it is helpful to keep them separate.

The simulation calculations have been carried out using the GEANT4 UCN-Monte Carlo code [21], which tracks UCN through a detailed model of the target-cell UCN spectrometer system, and used to extract estimates for the UCN extraction efficiency $\varepsilon_{\text {ext }}$ for the various sample temperatures. The detailed results will be considered together with the analysis in Sec. IV.

\section{RESULTS}

\section{A. UCN production in flow-through mode}

The main results from the experiment are the variation of UCN production with temperature of the ${ }^{2} \mathrm{H}_{2}$; these are shown in Figs. 4 and 5 as counts (corrected for background) per coulomb proton charge onto the SINQ target as a function of temperature.

At $8 \mathrm{~K}$, a neutron current of $I^{\mathrm{FM}}=(0.42 \pm 0.04) \mathrm{s}^{-1}$ has been measured for $1.2 \mathrm{~mA}$ proton beam onto the SINQ target. The data point at $85 \mathrm{~K}$ shown in Fig. 4 was measured for the evacuated target cell. Thus, without ${ }^{2} \mathrm{H}_{2}$ in the target, the measured UCN production is consistent with zero. All other data points are with ${ }^{2} \mathrm{H}_{2}$ in the target cell, above $24 \mathrm{~K}$ as gas, between 24 and $18.7 \mathrm{~K}$ liquid, and below $18.7 \mathrm{~K}$ solid. Data for two differently prepared crystals are included in Fig. 5, one was cooled slowly, the other one fast (see discussion in Sec. IV D).

\section{B. UCN storage mode}

The storage mode measurements were performed to unambiguously demonstrate that the produced neutrons

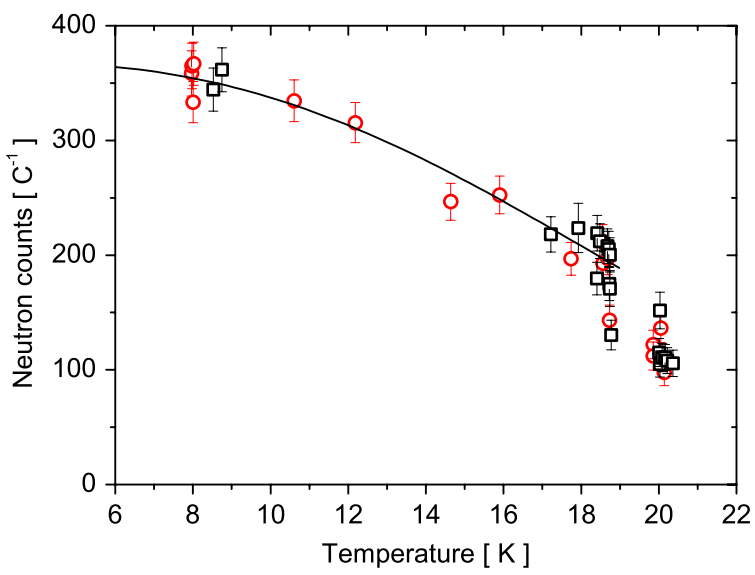

FIG. 5. (Color online) Same as Fig. 4 but zoomed in on the liquid and solid ${ }^{2} \mathrm{H}_{2}$ region. Circles are for the slowly cooled crystal; squares are for the rapidly cooled crystal. The solid line was obtained using the simulation for UCN extraction and taking into account the temperature dependences of both thermal upscattering losses and UCN production (see Sec. IV E).

actually were UCN and that quantitative agreement with the flow-through results exists so that the latter can be used to draw conclusions about UCN production. Storage mode measurements were more time consuming and were made only for the case of solid ${ }^{2} \mathrm{H}_{2}$ at $8 \mathrm{~K}$, which was the lowest accessible temperature and gave the highest flow-through rates. Figures 6 and 7 show examples for different storage times with UCN counts accumulated for 100 filling-storage-emptying cycles. The background has been subtracted from the data. Figure 8 shows the resulting UCN counts for four different storage times. An exponential fit to the data points gives a storage lifetime of $24 \pm 8 \mathrm{~s}$ and an extrapolation to $t=0$ of

$$
N_{\mathrm{UCN}}^{\mathrm{SM}}=0.21 \pm 0.03
$$

$\mathrm{UCN}$ in the bottle at equilibrium per storage cycle.

The value in (2) is consistent with the flow-through mode measurement. The flow-through mode measures the net neutron current through the bottle. The neutron spectrum in the flow-through mode (see below and Fig. 12) is rather wider than can be stored in the bottle, $v_{c}=6.9 \mathrm{~m} / \mathrm{s} ;(45 \pm 4) \%$ of the neutrons have $v \leqslant v_{c}$ (the hatched area in Fig. 12). Thus, closing the shutter UCN 2 (see Fig. 1) makes the UCN fraction of this the current, $I_{\mathrm{UCN}}=0.45(4) \times I^{\mathrm{FM}}$, filling the bottle. After a filling time $t_{f}$, the number of neutrons in the bottle is

$$
N_{\mathrm{UCN}}=I_{\mathrm{UCN}} \tau_{1}\left(1-e^{-t_{f} / \tau_{1}}\right),
$$

where $\tau_{1}$ is the UCN lifetime in the bottle with either shutter $\mathrm{UCN} 1$ or UCN 2 open. This time constant is dictated by the geometry of the bottle system. The symmetry of the system used suggests that the time constants for the arrangement shutter UCN 1 closed, UCN 2 open and the opposite arrangement are the same. Thus, $\tau_{1}$ can be extracted from the emptying curve of the storage measurements (compare Figs. 6 and 7) and found to be $\tau_{1}=0.97 \pm 0.25 \mathrm{~s}$. Therefore, the estimated number of UCN in the bottle per filling cycle by using the 


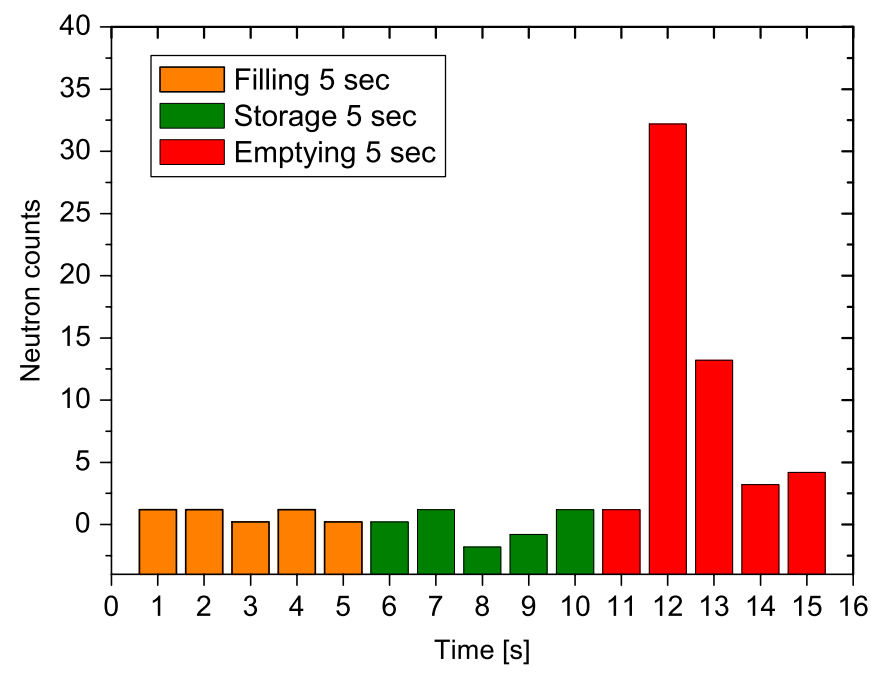

FIG. 6. (Color online) Background-subtracted time spectra for UCN counts during filling, storing, and emptying times of $5 \mathrm{~s}$ each.

flow-through data is

$$
N_{\mathrm{UCN}}^{\mathrm{FM}}=0.18 \pm 0.05
$$

\section{ANALYSIS AND DISCUSSION}

\section{A. General considerations}

In the flow-through mode, the neutron count rate $I$ is measured for a given incoming neutron flux $\Phi\left(E_{i}\right)$ and for various target conditions (gas, liquid, and solid at various temperatures, with ${ }^{2} \mathrm{H}_{2}$ density $\rho$ and illuminated target volume $V_{\mathrm{D}}$ ). Using subscripts $i$ and $f$ to denote values for initial and final states, we can write

$\frac{I}{\rho V_{\mathrm{D}}}=\iint \varepsilon_{\mathrm{rest}} \varepsilon_{\mathrm{tra}} \varepsilon_{\mathrm{ext}} \Phi\left(E_{i}\right) \sigma\left(E_{i} \rightarrow E_{f}\right) d E_{i} d E_{f}$

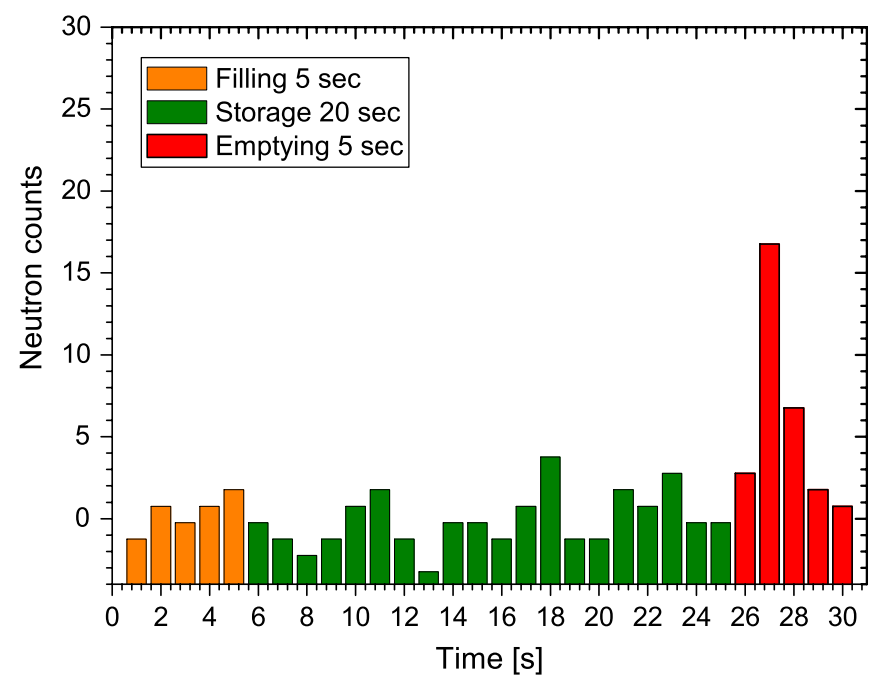

FIG. 7. (Color online) Background-subtracted time spectra for UCN counts during $5 \mathrm{~s}$ filling, $20 \mathrm{~s}$ storing, and $5 \mathrm{~s}$ emptying.

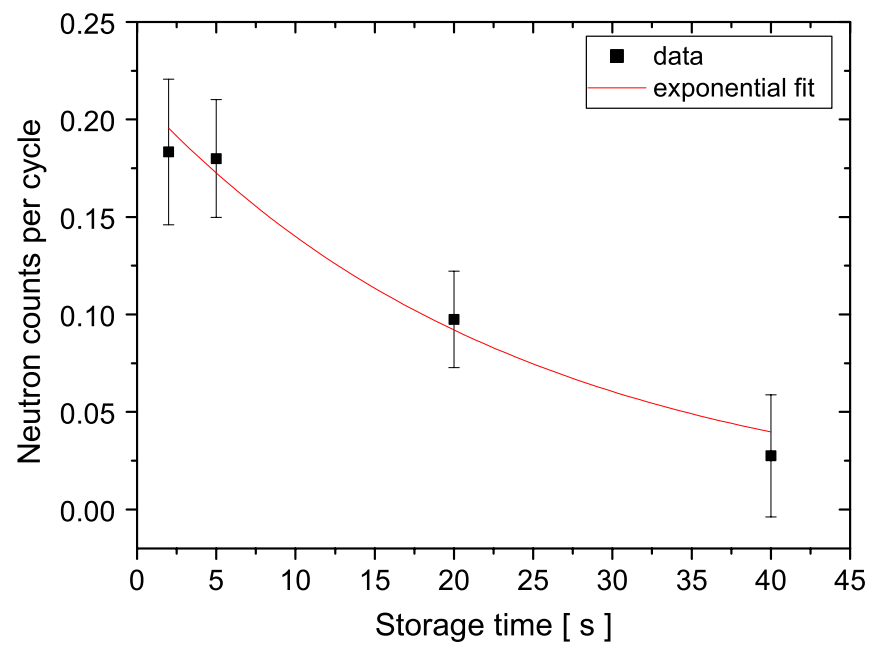

FIG. 8. (Color online) Decay of the UCN density in the bottle as a function of storage time. The four measured points are given along with an exponential fit. The storage lifetime is found to be $24 \pm 8 \mathrm{~s}$; the equilibrium population after filling is $0.21 \pm 0.03 \mathrm{UCN}$ per fill.

where $\sigma\left(E_{i} \rightarrow E_{f}\right)$ is the down-scattering cross section and three factors are introduced: $\varepsilon_{\text {rest }}$ for the efficiency of the actual detector and other loss factors outside the simulation (e.g., gaps between guide sections), $\varepsilon_{\text {tra }}$ the efficiency for transporting the neutrons through the UCN spectrometer, and $\varepsilon_{\text {ext }}$ the efficiency for the transport of the neutrons from their production position to the start of the spectrometer. These three factors depend on final energy $E_{f}$ and are obtained from a simulation of the experiment and from use of measurements with gaseous ${ }^{2} \mathrm{H}_{2}$. The experiment is not set up to measure the influence of the incident energy $E_{i}$, and $\sigma$ is replaced by an average cross section for producing neutrons of energy $E_{f}, \bar{\sigma}\left(E_{f}\right)$, which is proportional to $\sqrt{E_{f}}$. Equation (5) may be rewritten as

$$
\frac{I}{\rho V_{\mathrm{D}}}=\Phi_{\mathrm{CN}} \int \varepsilon_{\mathrm{rest}} \varepsilon_{\mathrm{tra}} \varepsilon_{\mathrm{ext}} \bar{\sigma}\left(E_{f}\right) d E_{f}
$$

The dependence of $\varepsilon_{\text {rest }}$ on $E_{f}$ will be weak and can be separated and replaced by an average $\bar{\varepsilon}_{\text {rest }}$. It is also sometimes more convenient to work with velocity as the dependent variable. The velocity dependence of $\bar{\sigma}\left(v_{f}\right)=\bar{\sigma}^{\mathrm{CN} \rightarrow \mathrm{UCN}} f(v)$ is known, $f(v) \sim v^{2}$, so that Equation (6) can be written as

$$
\frac{I}{\rho V_{\mathrm{D}}}=\Phi_{\mathrm{CN}} \bar{\varepsilon}_{\mathrm{rest}} \bar{\sigma}^{\mathrm{CN} \rightarrow \mathrm{UCN}} \int P(v) d v,
$$

where

$$
P(v)=f(v) \varepsilon_{\mathrm{ext}}(v) \varepsilon_{\mathrm{tra}}(v)
$$

with the normalization condition

$$
\int_{v_{1}}^{v_{2}} f(v) d v=1
$$

where $v_{1}$ and $v_{2}$ are the velocities in the target material that lead to UCN storable in the bottle. 


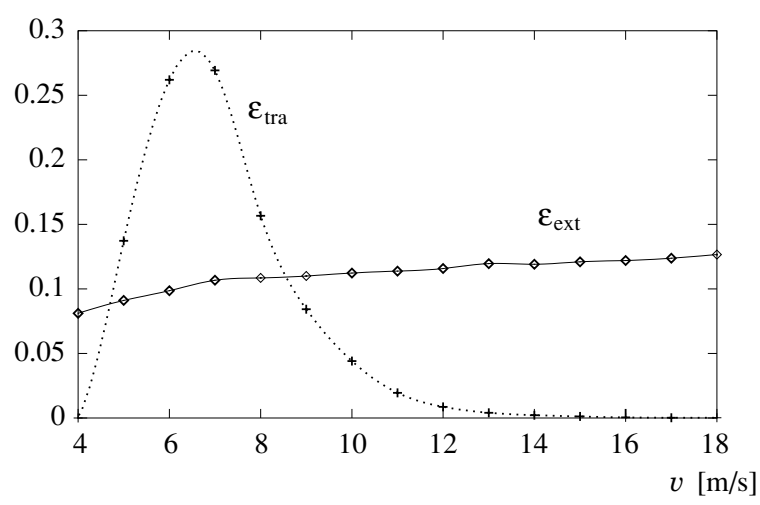

FIG. 9. Simulated efficiencies for extraction $\varepsilon_{\mathrm{ext}}$ and transport $\varepsilon_{\text {tra }}$ as functions of neutron velocity inside the ${ }^{2} \mathrm{H}_{2}$ gas target at $24 \mathrm{~K}$. Ultracold neutrons with velocities below $4 \mathrm{~m} / \mathrm{s}$ in the gas target cannot reach the detector.

\section{B. UCN production by ${ }^{2} \mathrm{H}_{2}$ gas and the extraction of values for $\varepsilon_{\text {tra }}$ and $\bar{\varepsilon}_{\text {rest }}$}

From the general considerations set out in Sec. IIC, the main open parameter stopping the determination of $\varepsilon_{\text {tra }}$ is the diffuse-scattering fraction at the guide wall. A value can be assigned that together with $\bar{\varepsilon}_{\text {rest }}$ matches experiment and simulation calculation. It is clear that unique values cannot be obtained. However, limiting values to those that are physically reasonable, e.g., a diffuse-scattering fraction of more than 3\% would require $\bar{\varepsilon}_{\text {rest }}$ to be greater than 1 , yields an indicative value for the diffuse-scattering fraction. We finally set the diffuse-scattering fraction in the simulation to $1 \%$ as this leads to a reasonable numerical value for $\bar{\varepsilon}_{\text {rest }}$ [see Eq. (13) below].

The individual calculated values for $\varepsilon_{\text {tra }}$ and $\varepsilon_{\text {ext }}$ obtained from the gas-target simulation are shown in Fig. 9, and the values for the efficiency function $P(v)$ are displayed in Fig. 10. The neutrons contributing to the UCN stored in the bottle lie in the energy range 100 to $350 \mathrm{neV}$ in the gas,

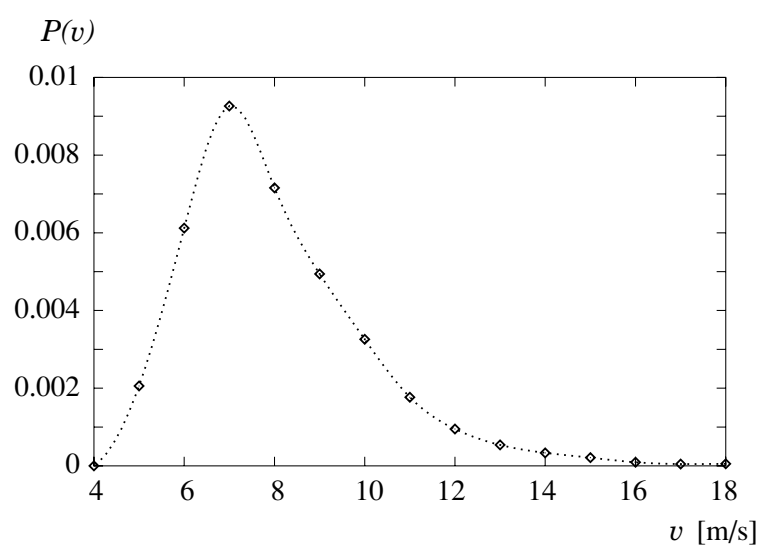

FIG. 10. The product $P(v)$ of the simulated efficiencies $\varepsilon_{\text {ext }}, \varepsilon_{\text {tra }}$ (see Fig. 9) and the velocity distribution $f(v)$ is displayed [see Eq. (8)] for the ${ }^{2} \mathrm{H}_{2}$ gas target at $24 \mathrm{~K}$ as a function of neutron velocity inside the target. The normalization condition for $f(v)$ is explained in the text.
TABLE I. Measured numbers of UCN (flow-through mode) for a proton beam charge of $1 \mathrm{C}$ on the SINQ spallation target for different target conditions. The measurements were made while warming up the target, and the temperatures given are average values. The ${ }^{2} \mathrm{H}_{2}$ number densities $\rho$ have been calculated for the van der Waals gas parameters of Ref. [22]. Within the experimental uncertainties, $I / \rho$ is constant.

\begin{tabular}{lccc}
\hline \hline$T(\mathrm{~K})$ & $\rho\left(\mathrm{cm}^{-3}\right)$ & $I\left(\mathrm{C}^{-1}\right)$ & $I / \rho\left(\mathrm{C}^{-1} \mathrm{~cm}^{3}\right)$ \\
\hline $24^{*}$ & $3.19 \times 10^{20}$ & $104 \pm 12$ & $3.3(4) \times 10^{-19}$ \\
48 & $2.08 \times 10^{20}$ & $71 \pm 10$ & $3.4(5) \times 10^{-19}$ \\
64 & $1.54 \times 10^{20}$ & $50 \pm 10$ & $3.2(6) \times 10^{-19}$ \\
75 & $1.31 \times 10^{20}$ & $45 \pm 9$ & $3.4(7) \times 10^{-19}$ \\
85 & Evacuated & $-2 \pm 5$ & \\
\hline \hline
\end{tabular}

*For the $24 \mathrm{~K}$ data, it is not clear whether the target might have contained some liquid ${ }^{2} \mathrm{H}_{2}$.

so that

$$
P_{\mathrm{int}}^{\mathrm{gas}}=\int_{4.37 \mathrm{~m} / \mathrm{s}}^{8.18 \mathrm{~m} / \mathrm{s}} P(v) d v=0.037 .
$$

The detailed results of the simulation show that the UCN extraction efficiency is almost independent of temperature, as the variation of density and neutron up scattering cross section virtually compensate one another; the measured values for the gas target (collected in Table I) are consistent with this.

We use the YK model (see Sec. IIC) to calculate the down-scattering cross sections $\sigma_{\mathrm{YK}}$ of neutrons of initial energy $E_{i}$ into the UCN region. The final energies have been set to the interval $100-350 \mathrm{neV}$, which is consistent with the energy range for neutrons in the gas that can be stored in the bottle at $1 \mathrm{~m}$ height.

$$
\begin{aligned}
\sigma_{\mathrm{YK}}\left(E_{i} \rightarrow E_{\mathrm{UCN}}\right) & =\int_{100 \mathrm{neV}}^{350 \mathrm{neV}} \frac{d \sigma_{\mathrm{YK}}}{d E_{f}}\left(E_{i}\right) d E_{f} . \\
\sigma_{\mathrm{YK}}^{\mathrm{CN} \rightarrow \mathrm{UCN}} & =\int \Phi\left(E_{i}\right) \sigma_{\mathrm{YK}}\left(E_{i} \rightarrow E_{\mathrm{UCN}}\right) d E_{i} .
\end{aligned}
$$

The calculated down-scattering cross sections, $\sigma_{\mathrm{YK}}^{\mathrm{CN} \rightarrow \mathrm{UCN}}$, together with other derived parameters relevant to estimation of $\bar{\varepsilon}_{\text {rest }}$ are collected in Table II. The (positive) uncertainties attached to the values of $\sigma_{\mathrm{YK}}^{\mathrm{CN} \rightarrow \mathrm{UCN}}$ in Table II come from an estimation of the contribution of lower energy neutrons (below $0.2 \mathrm{meV}$, see Fig. 2) in the FUNSPIN spectrum. This estimate was made using an extrapolation on the basis that the source spectrum for the FUNSPIN guide is Maxwellian. We note that this adds at most a few percent additional uncertainty, which is small compared to the statistical uncertainty of the gas measurement. The four individual determinations of $\bar{\varepsilon}_{\text {rest }}$ in the last column are statistically consistent and give an overall average value of

$$
\bar{\varepsilon}_{\text {rest }}=0.74 \pm 0.06 \pm 0.16 \text {. }
$$

This value is affected by the $( \pm 0.16)$ systematic uncertainty of the cold neutron flux determination [see Eq. (1)]. However, not affected by this systematics is the product

$$
\bar{\varepsilon}_{\text {rest }} \Phi_{\mathrm{CN}}=(3.33 \pm 0.27) \times 10^{10} \mathrm{C}^{-1} .
$$


TABLE II. Calculated down-scattering (single-scattering) cross sections per molecule averaged over the incoming cold neutron spectrum (see Fig. 2) and various ${ }^{2} \mathrm{H}_{2}$ gas temperatures. See text for an explanation of the uncertainties in the second column; $E_{\mathrm{UCN}}=100 \ldots 350 \mathrm{neV} ; V=45 \mathrm{~cm}^{3} ; R_{\mathrm{gas}}=\sigma_{\mathrm{YK}}^{\mathrm{CN} \rightarrow \mathrm{UCN}} \rho ; P_{\mathrm{UCN}}=$ $\Phi_{\mathrm{CN}} R_{\text {gas }} V ; P_{\text {int }}=0.037 ; \bar{\varepsilon}_{\text {rest }}=I /\left(P_{\text {int }} P_{\mathrm{UCN}}\right)$. The attached errors on $\bar{\varepsilon}_{\text {rest }}$ concern the last digits and are for the statistical uncertainty (first) and the systematics (second) coming from the measurement of $\Phi_{\mathrm{CN}}$. The values in the last column are not affected by this systematic uncertainty.

\begin{tabular}{lcccccc}
\hline \hline $\mathrm{T}$ & $\begin{array}{c}\sigma_{\mathrm{YK} \rightarrow \mathrm{UCN}}^{\mathrm{CN}} \\
\left(10^{-30} \mathrm{~cm}^{2}\right)\end{array}$ & $\begin{array}{c}R_{\text {gas }} \\
\left(10^{-9} \mathrm{~cm}^{-1}\right)\end{array}$ & $\begin{array}{c}P_{\mathrm{UCN}} \\
\left(\mathrm{C}^{-1}\right)\end{array}$ & $\begin{array}{c}P_{\text {int }} P_{\mathrm{UCN}} \\
\left(\mathrm{C}^{-1}\right)\end{array}$ & $\bar{\varepsilon}_{\text {rest }}$ & $\begin{array}{c}\bar{\varepsilon}_{\text {rest }} \Phi_{\mathrm{CN}} \\
\left(10^{10} \mathrm{C}^{-1}\right)\end{array}$ \\
\hline 24 & $6.63(+0.22)$ & 2.11 & 4273 & 158 & $0.66(8)(15)$ & $2.96(36)$ \\
48 & $6.01(+0.16)$ & 1.25 & 2531 & 94 & $0.76(11)(17)$ & $3.41(49)$ \\
64 & $5.66(+0.15)$ & 0.87 & 1762 & 65 & $0.77(15)(17)$ & $3.45(67)$ \\
75 & $5.42(+0.14)$ & 0.71 & 1438 & 53 & $0.85(17)(19)$ & $3.81(76)$ \\
\hline \hline
\end{tabular}

\section{UCN production by liquid ${ }^{2} \mathrm{H}_{2}$}

In the case of the liquid ${ }^{2} \mathrm{H}_{2}$, the high density means that both the incident cold neutrons and the produced UCN will be significantly attenuated over the length of the target. These effects are taken into account in the simulation of $\varepsilon_{\text {ext }}$. The CN flux distribution within the liquid ${ }^{2} \mathrm{H}_{2}$ is calculated using the code MCNPX [23]; the measured incoming spectrum (see Fig. 2) and the liquid ortho- ${ }^{2} \mathrm{H}_{2}$ scattering law of Bernnat et al. [24] are used, and a selection of flux spectra from the calculation is displayed in Fig. 11. The expected cooling of the incident $\mathrm{CN}$ spectrum may be seen; but as UCN production cross sections averaged over the incident spectrum are used throughout (Sec. IV A), this effect is not included in the simulation. An attenuation factor $\alpha_{\text {att }}=0.65 \pm 0.10$ is deduced for which $\alpha_{\text {att }} \Phi_{\mathrm{CN}}$ corresponds to the average cold neutron flux over the target. The assigned systematic uncertainty accounts for limitations in the simulation of the target cell itself and its surroundings.

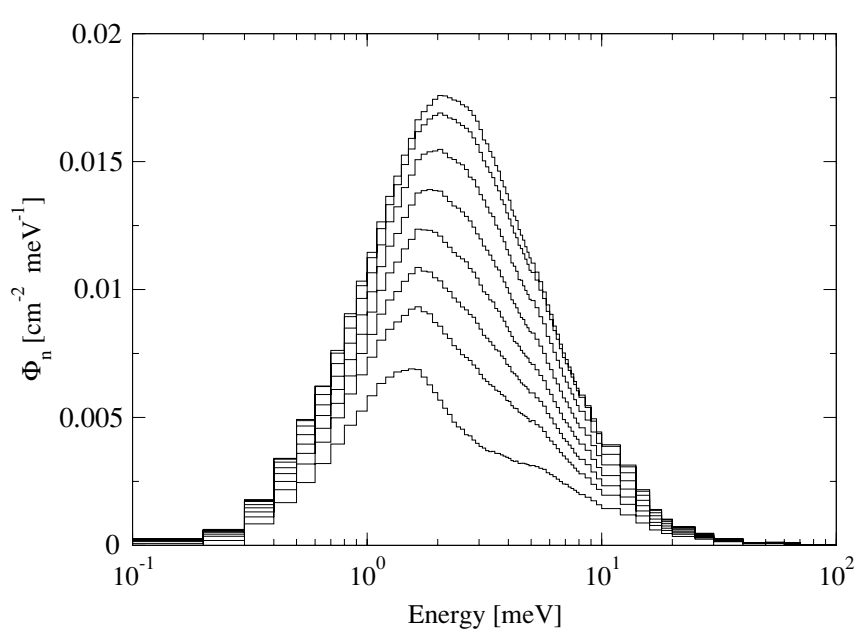

FIG. 11. MCNPX [23] simulation of the attenuation and spectral change of the cold neutron flux per incident source particle in liquid ${ }^{2} \mathrm{H}_{2}$. The spectra displayed are averages over $10 \mathrm{~mm}$ diameter, $5 \mathrm{~mm}$ long cylinders on the beam axis. The intensity varies systematically with distance, with the highest values by the entrance window and the lowest by the UCN exit window. The simulation used the IKE Stuttgart scattering kernel [24].
The simulation to obtain $P(v)$, see Fig. 12, uses the calculated spatial variation of cold flux within the liquid together with an isotropic UCN spectrum $f(v) \sim v^{2}$ to define the source and the recently measured liquid ${ }^{2} \mathrm{H}_{2}$ cross sections [15]. Taking a material potential for liquid ${ }^{2} \mathrm{H}_{2}$ of $90 \mathrm{neV}$ (i.e., the neutron energy range for producing stored neutrons in the bottle is now 10 to $260 \mathrm{neV}$ ), then

$$
P_{\text {int }}^{\text {liquid }}=\int_{1.38 \mathrm{~m} / \mathrm{s}}^{7.05 \mathrm{~m} / \mathrm{s}} P(v) d v=0.007 \pm 0.001
$$

The systematic uncertainty has been determined from the propagation of the estimated errors in the primary spatial distribution.

The neutron rate for liquid ${ }^{2} \mathrm{H}_{2}$ at $20 \mathrm{~K}$ (see Fig. 5) is measured as $I=(115 \pm 10) \mathrm{C}^{-1}$. Using the value for $\bar{\varepsilon}_{\text {rest }} \Phi_{\mathrm{CN}}=(3.33 \pm 0.27) \times 10^{10} \mathrm{C}^{-1}$ determined from the gas results [Sec. IV B, Eq. (14)] and taking the liquid density from [25], then

$$
\begin{aligned}
\sigma_{\text {liquid,20K }}^{\mathrm{CN} \rightarrow \mathrm{UCN}} & =\frac{I}{\alpha_{\text {att }} \Phi_{\mathrm{CN}} \rho V P_{\text {int }}^{\text {liquid }} \bar{\varepsilon}_{\text {rest }}} \\
& =(6.6 \pm 1.6) \times 10^{-31} \mathrm{~cm}^{2}
\end{aligned}
$$

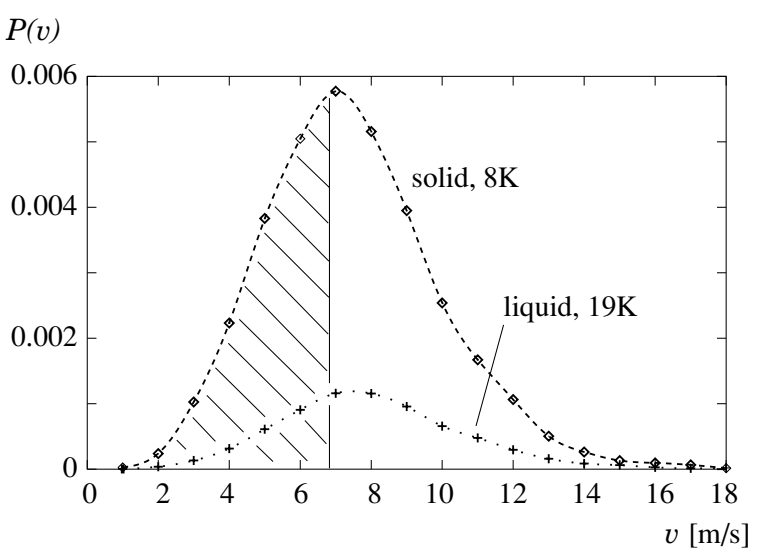

FIG. 12. The product $P(v)$ of the simulated efficiencies $\varepsilon_{\mathrm{ext}}$ and $\varepsilon_{\text {tra }}$ and the velocity distribution $f(v)$ is displayed [see Eq. (8)] for liquid and solid ${ }^{2} \mathrm{H}_{2}$, respectively. The different normalization conditions for $f(v)$ are explained in the text. 
and

$$
\begin{aligned}
R_{\mathrm{liquid}, 20 \mathrm{~K}} & =\sigma_{\mathrm{liquid}, 20 \mathrm{~K}}^{\mathrm{CN} \rightarrow \mathrm{UCN}} \rho \\
& =(1.70 \pm 0.41) \times 10^{-8} \mathrm{~cm}^{-1} .
\end{aligned}
$$

\section{UCN production from solid ${ }^{2} \mathbf{H}_{2}$}

The method used for extraction of UCN production cross sections for solid ${ }^{2} \mathrm{H}_{2}$ is completely analogous to that used for the liquid. The $P(v)$ distribution from the simulation is included in Fig. 12. The energy range for the source neutrons is 0 to $250 \mathrm{neV}$ in this case (the material potential of the solid is roughly $100 \mathrm{neV}$ ) and

$$
P_{\text {int }}^{\text {solid }}=\int P(v) d v=0.034 \pm 0.004 .
$$

The uncertainty is dominated by the effect of variations in the UCN scattering and loss cross sections on the extraction efficiency $\varepsilon_{\text {ext }}$; because the losses are small, comparable to those in the case of gas targets, this turns out to be quite a weak effect.

We detected neutron rates of $I=(360 \pm 10) \mathrm{C}^{-1}$ in solid ${ }^{2} \mathrm{H}_{2}$ at $8 \mathrm{~K}$, see Fig. 5. Again taking $\bar{\varepsilon}_{\text {rest }} \Phi_{\mathrm{CN}}$ from Eq. (14), using the same cold neutron attenuation factor $\alpha_{\text {att }}$ as for liquid and taking the solid density from [25], we then obtain

$$
\begin{aligned}
\sigma_{\text {solid, 8K }}^{\mathrm{CN} \rightarrow \mathrm{UCN}} & =\frac{I}{\alpha_{\text {att }} \Phi_{\mathrm{CN}} \rho V P_{\mathrm{int}}^{\text {solid }} \bar{\varepsilon}_{\text {rest }}} \\
& =(3.7 \pm 0.8) \times 10^{-31} \mathrm{~cm}^{2},
\end{aligned}
$$

and

$$
\begin{aligned}
R_{\text {solid }, 8 \mathrm{~K}} & =\sigma_{\text {solid }, 8 \mathrm{~K}}^{\mathrm{CN} \rightarrow \mathrm{UCN}} \rho \\
& =(1.11 \pm 0.23) \times 10^{-8} \mathrm{~cm}^{-1} .
\end{aligned}
$$

In the experiment, we included measurements to check that the treatment of solid ${ }^{2} \mathrm{H}_{2}$ does not strongly influence the UCN yield. Two different ${ }^{2} \mathrm{H}_{2}$ crystals were used; the first one was grown and annealed close to the triple point over $8 \mathrm{~h}$ and then cooled down slowly to $8 \mathrm{~K}$ over another $8 \mathrm{~h}$; the second one was grown at the triple point and then cooled rapidly from $17 \mathrm{~K}$ to $8 \mathrm{~K}$ within $15-20 \mathrm{~min}$. The UCN production by both crystals was the same within the statistical uncertainties. Data from both crystals are included in Fig. 5.

\section{E. Discussion}

The observation that the differently prepared crystals produce equally high UCN rates allows one to conclude that UCN extraction at $8 \mathrm{~K}$, in the geometry of the present experiment, is not strongly influenced by specific details of the crystal, and this should be the case when the average time the UCN spend in the ${ }^{2} \mathrm{H}_{2}$ before extraction becomes small in comparison with their lifetime in the solid. For solid ${ }^{2} \mathrm{H}_{2}$ at $8 \mathrm{~K}$ used in the present experiment, the estimated UCN lifetime is about $24 \mathrm{~ms}$, dominated by thermal up scattering (40 ms lifetime) and influenced by the para up scattering (about $100 \mathrm{~ms})[26]$ and the neutron-deuteron absorption $(150 \mathrm{~ms})$. (Hydrogen contamination of the target was negligible in our case.) Neutrons with velocities of $5 \mathrm{~m} / \mathrm{s}$ travel $12 \mathrm{~cm}$ in $24 \mathrm{~ms}$, which is much larger than the target dimensions in our experiment. For solid ${ }^{2} \mathrm{H}_{2}$ at $8 \mathrm{~K}$ under comparable conditions (e.g. $98 \%$ ortho concentration), UCN lifetimes of about $20 \mathrm{~ms}$ have been measured [12].

It is instructive to compare our UCN production cross section, Eq. (20), with predictions $[9,10]$. These calculations were performed using the incoherent approximation, single phonon exchange, and one of two models for the phonon spectrum, the Debye model (A) or a "realistic" spectrum (B) [10]. To make them comparable, the equilibrium UCN densities presented in [10] should be divided by the UCN lifetime due to absorption $(150 \mathrm{~ms})$ and by a factor of 0.75 to remove their correction for the ${ }^{2} \mathrm{H}_{2}$ potential. We extract from [10] values for solid ${ }^{2} \mathrm{H}_{2}$ at $5 \mathrm{~K}$ and for a Maxwellian neutron spectrum with $T_{n} \approx 25 \mathrm{~K}$ (being not particularly sensitive to either and thus appropriate for a rough comparison with $8 \mathrm{~K}$ and FUNSPIN):

$$
R_{\mathrm{A}}=1.1 \times 10^{-8} \mathrm{~cm}^{-1}, \quad R_{\mathrm{B}}=1.3 \times 10^{-8} \mathrm{~cm}^{-1} .
$$

Together with the authors of [10], one concludes that the specific form of the phonon spectrum is not of great importance for the UCN production. They further argue that neglecting the influence of molecular effects in their treatment resulted in an average $20 \%$ overestimation of the cross sections. At the same time, the consideration of multiple phonon creation will raise the cross sections by a similar amount. This suggests that our experimental result is in agreement with the predicted cross sections, even that based on the simple Debye spectrum.

The temperature dependence of the measured UCN current from solid ${ }^{2} \mathrm{H}_{2}$, see Fig. 5, can in principle be treated within the same model and understood as the balance between the temperature dependence of thermal up scattering and UCN production. However, one finds that limiting the treatment of UCN production to the creation of only one single phonon does not properly describe the system. The inclusion of multiple phonon production in the down-scattering process and, thus, a temperature-dependent UCN source leads to an agreement of the measured data and the simulation of the extraction (see curve in Fig. 5). The major significant temperature dependence in down scattering, in case of the FUNSPIN spectrum, is obtained already from the two-phonon creation. It is worth mentioning that almost the same curve is obtained by fitting a simple product of production and loss to the data with only one scale factor $N_{0}$ and one more parameter $d$ for the mean UCN track length in the target:

$$
N(T)=N_{0} \frac{\sigma_{\text {down }}(T)}{\sigma_{\text {down }}(8 \mathrm{~K})} \exp \left[-\rho \sigma_{\text {up }}(T) d\right] .
$$

Here we calculate $\sigma_{\text {up }}(T)$ in accordance with, e.g. [26], and $\sigma_{\text {down }}(T)$ along the lines of [27] and obtain from the fit $d \approx 2 \mathrm{~cm}$ for the path length, in agreement with the results from the simulation for the average UCN velocity of $7 \mathrm{~m} / \mathrm{s}$ (see Fig. 12).

It is important to note that the observed increase in extracted UCN between 20 and $8 \mathrm{~K}$ (see Fig. 5) cannot directly be compared with the "gain factors" as reported in $[8,11]$. In the present experiment, the extraction of production cross sections 
is straightforward because the incoming cold neutron spectrum does not change significantly with target temperature, while the incoming spectrum is strongly affected in $[8,11]$ due to the sample being the cold moderator at the same time. Also, the geometries and sizes of the cold sources influence strongly the observed experimental gain as can be seen when comparing [8] and [11].

Next, the result from above for the UCN production is compared with predictions for the UCN density in the source, which is currently under construction at PSI [14]. With the $2 \mathrm{~mA}$ proton beam onto the $\mathrm{Pb}$ spallation target, a cold neutron flux of about $2.6 \times 10^{13} \mathrm{~cm}^{-2} \mathrm{~s}^{-1}$ in the relevant region of the deuterium moderator will produce $2.9 \times 10^{5} \mathrm{~cm}^{-3} \mathrm{~s}^{-1}$ UCN [see Eq. (20)]. The lifetime of the UCN in the converter depends on the temperature. During a proton beam pulse of $4 s(8 s)$ the temperature of the UCN-producing region of the ${ }^{2} \mathrm{H}_{2}$ is calculated to rise from 5 to $8 \mathrm{~K}(10 \mathrm{~K})$ while the UCN density reaches about $50 \%(80 \%)$ of the saturation value. Thus, conservatively assuming a $4 \mathrm{~s}$ long proton pulse and a constant lifetime of $24 \mathrm{~ms}$ for $\mathrm{UCN}$ in solid ${ }^{2} \mathrm{H}_{2}$ (at $8 \mathrm{~K}$ as in this experiment and at the end of the $4 \mathrm{~s}$ pulse), one calculates a density of about $3500 \mathrm{~cm}^{-3}$ in the UCN storage vessel. This is in good agreement with the predicted UCN density [14]. Proton pulses exceeding $8 \mathrm{~s}$ are not foreseen because of the heat load on the moderator; a practical $8 \mathrm{~s}$ limit of the proton pulse length has been implemented by the layout of the power supply of the kicker magnet in the proton beam line [28]. The optimum (potentially shorter) pulse length will be determined experimentally by analyzing the source performance with the boundary condition of an overall $1 \%$ duty cycle (a limit imposed by radiation protection considerations). It is not expected that the converter performance will deteriorate under irradiation besides the increase in thermal up scattering due to increased temperature. The ortho-deuterium concentration will be affected only slightly but rather improved [29,30], and recently it was shown that thermal cycling between 5 and $10 \mathrm{~K}$ does not deteriorate the UCN transmission of carefully prepared ${ }^{2} \mathrm{H}_{2}$ crystals [16].

\section{SUMMARY}

We have presented the results of a UCN production experiment using ${ }^{2} \mathrm{H}_{2}$ as a target for a cold neutron beam.
Ultracold neutrons were produced, stored, and detected. The background level in the experiment was sufficiently low to also detect UCN without storage. UCN signal-to-background ratios of up to 40:1 were obtained with 5\% statistical accuracy in measurements lasting less than $1 / 2 \mathrm{~h}$, depending on the conditions of the target.

The comparison of the measured and calculated UCN production on gaseous ${ }^{2} \mathrm{H}_{2}$ was used to calibrate the simulated target extraction and transport efficiencies of the experimental apparatus. Absolute production rates and cross sections for liquid and solid ${ }^{2} \mathrm{H}_{2}$ were extracted from the data. The value for low-temperature solid ${ }^{2} \mathrm{H}_{2}$ agreed with the cross section calculated using the simple Debye model and the incoherent approximation. The temperature dependence of the measured UCN rate can be understood in terms of the variations in balance between the actual UCN production (including multiple-phonon excitation) and thermal up scattering.

In previous UCN production experiments [11-13], the incoming neutron spectra were not known in detail. Consistent results were obtained by combining calculated cold neutron spectra and UCN production models along the lines of $[9,10,27]$ and as discussed above. The present work validated the UCN production cross sections. Based on that, we corroborate the UCN density predictions for the new high-intensity source currently under construction at PSI.

\section{ACKNOWLEDGMENTS}

This work was performed at the FUNSPIN beamline of the Swiss spallation neutron source SINQ, Paul Scherrer Institut, Villigen, Switzerland. We are grateful to the machine and beamline groups, as well as to various technical support services and workshops whose outstanding efforts made these experiments possible. We are especially grateful for the support of K. Kohlik in layout and design of the equipment. We acknowledge W. Arrigoni and P. Schurter for manufacturing and assembling the cryostat and target cell, M. Horisberger for sputtering the Fe and Ni mirrors, PNPI for providing the Be coating of the storage tube, and G. Frey and M. Luethy for the gold foil activation analysis. We are also grateful to A. Kozela, J. Pulut, and J. Zejma for helping with specific details of the FUNSPIN beam and area. This work was supported by the Swiss National Science Foundation (Grant No. 2100-067840).
[1] V. K. Ignatovich, Ultracold Neutrons (Clarendon Press, Oxford, 1990).

[2] R. Golub, D. J. Richardson, and S. K. Lamoreaux, Ultra-cold Neutrons, (Hilger, Bristol, 1991).

[3] A. Steyerl et al., Phys. Lett. A116, 347 (1986).

[4] R. Golub and J. M. Pendlebury, Phys. Lett. A62, 337 (1977).

[5] C. R. Brome et al., Phys. Rev. C 63, 055502 (2001).

[6] Y. Masuda, T. Kitagaki, K. Hatanaka, M. Higuchi, S. Ishimoto, Y. Kiyanagi, K. Morimoto, S. Muto, and M. Yoshimura, Phys. Rev. Lett. 89, 284801 (2002).

[7] C. A. Baker et al., Phys. Lett. A308, 67 (2003).
[8] I. S. Altarev et al., Phys. Lett. A80, 413 (1980).

[9] R. Golub and K. Böning, Z. Phys. B 51, 95 (1983).

[10] Z.-Ch. Yu, S. S. Malik, and R. Golub, Z. Phys. B 62, 137 (1986).

[11] A. Serebrov et al., Nucl. Instrum. Methods A 440, 658 (2000).

[12] C. L. Morris, J. M. Anaya, T. J. Bowles, B. W. Filippone, P. Geltenbort, R. E. Hill, M. Hino, S. Hoedl, G. E. Hogan, T. M. Ito, T. Kawai, K. Kirch, S. K. Lamoreaux, C.-Y. Liu, M. Makela, L. J. Marek, J. W. Martin, R. N. Mortensen, A. Pichlmaier, A. Saunders, S. J. Seestrom, D. Smith, W. Teasdale, B. Tipton, M. Utsuro, A. R. Young, and J. Yuan, Phys. Rev. Lett. 89, 272501 (2002).

[13] A. Saunders et al., Phys. Lett. B593, 55 (2004). 
[14] http://ucn.web.psi.ch/

[15] F. Atchison et al., accepted for publication in Phys. Rev. Lett.

[16] F. Atchison et al. (will be submitted to Phys. Rev. C).

[17] J. Zejma et al., Nucl. Instrum. Methods A 539, 622 (2005).

[18] K. Bodek et al., Nucl. Instrum. Methods A 533, 491 (2004).

[19] J. A. Young and J. U. Koppel, Phys. Rev. 135, A603 (1964).

[20] J. U. Koppel and J. A. Young, Nukleonik 8, 40 (1966).

[21] F. Atchison, T. Bryś, M. Daum, P. Fierlinger, A. Fomin, R. Henneck, K. Kirch, M. Kuźniak, and A. Pichlmaier (submitted to Nucl. Instrum. Methods A).

[22] K. G. McLennan and E. MacA. Gray, Meas. Sci. Technol. 15, 211 (2004).

[23] L. S. Waters et al., MCNPX User's Manual Version 2.4.0, LA-CP-02-408, 2002.

[24] W. Bernnat, J. Keinert, and M. Mattes, in Proceedings of the 6th International Workshop on Advanced Cold Moderators, For chungszentrum Jülich Matter and Materials 20, edited by H. Conrad and Jülich 2004, p. 9.

[25] P. C. Souers, Hydrogen Properties for Fusion Energy (University of California, Berkeley, 1986).

[26] C.-Y. Liu, A. R. Young, and S. K. Lamoreaux, Phys. Rev. B 62, R3581 (2000).

[27] V. F. Turchin, Slow Neutrons (Israel Program for Scientific Translations, Jerusalem, 1965).

[28] D. Anicic et al., A fast kicker magnet for the PSI $600 \mathrm{MeV}$ proton beam to the PSI ultracold neutron source, Nucl. Instrum. Methods A 541, 598 (2005).

[29] F. Atchison et al., Phys. Rev. B 68, 094114 (2003).

[30] G. W. Collins, E. M. Fearon, E. R. Mapoles, R. T. Tsugawa, P. C. Souers, and P. A. Fedders, Phys. Rev. B 44, 6598 (1991). 